\title{
Postpartum Weight Retention Risk Factors in a Taiwanese Cohort Study
}

\author{
Hsin-Hui Shao Lee-Ching Hwang Jian-Pei Huang Hsin-Yin Hsu \\ MacKay Memorial Hospital, Taipei City, Taiwan
}

\section{Keywords}

Postpartum weight retention · Gestational weight gain · Obesity

\begin{abstract}
Objective: Excess postpartum weight retention (PPWR) is related to long-term weight gain. Therefore, this study was conducted to identify the risk factors for PPWR to provide guidance for preventive strategies. Methods: This cohort study surveyed 461 women who gave birth at a medical center between March 2014 and March 2016. The participants completed a questionnaire within 1 month of delivery, and their 6 -month postpartum weight was tracked. $\boldsymbol{R e}$ sults: The results showed that the mean pre-pregnancy BMI was $21.4 \pm 3.3 \mathrm{~kg} / \mathrm{m}^{2}$, and the mean gestational weight gain (GWG) was $12.8 \pm 4.1 \mathrm{~kg}$. The mean PPWR was $4.6 \pm 3.5 \mathrm{~kg}$ at 1 month and $2.1 \pm 3.3 \mathrm{~kg}$ at 6 months. Multivariate analysis revealed that GWG (adjusted OR: 1.92 (1.70-2.17)), pre-pregnancy BMI (adjusted OR: 0.85 (0.77-0.94)), and exclusive breastfeeding (adjusted OR: $0.55(0.32-0.94))$ were significantly correlated with a 1-month PPWR higher than the median value. In addition, GWG (adjusted OR: 1.30 (1.22-1.39)) and exclusive breastfeeding (adjusted OR: $0.37(0.24-0.58)$ ) were significantly correlated with a 6-month PPWR higher than the median value. Conclusion: Our findings indicate that the key to reducing PPWR is to control GWG and engage in exclusive breastfeeding.
\end{abstract}

(C) 2018 The Author(s)

Published by S. Karger GmbH, Freiburg

\section{Introduction}

The worldwide prevalence of overweight and obesity has been rising steadily since the 1980s. Obesity increases the risk of chronic diseases such as diabetes mellitus, cardiovascular disease, and several types of cancer [1]. Moreover, it has been found to have a significant impact on mortality [2].For women, changes in body weight occur naturally at certain life 
stages including pregnancy, postpartum, and menopause periods; all of these are critical stages during which women are prone to obesity [3]. A long-term follow-up study of weight development during and after pregnancy showed that women who gained excess weight within 15 years had retained more weight within the first year postpartum [4].

A cohort study in the US showed that approximately $75 \%$ of women were unable to return to their pre-pregnancy weight 1 year postpartum [5]. Another cohort study in Brazil reported a mean postpartum weight retention (PPWR) of $4.8 \mathrm{~kg}$ at 6 months [6]. A review article targeting women in Asia reported that the average 6-month PPWR in various countries ranges from 1.6 to $4.1 \mathrm{~kg}$ [7], and two studies in Taiwan both reported a 6-month PPWR of approximately $2.4 \mathrm{~kg}[8,9]$. However, few studies have investigated PPWR and its related factors in Taiwan.

Gestational weight gain (GWG) has been recognized as a major risk factor for PPWR, with numerous studies demonstrating a positive correlation between excessive GWG and PPWR [4--13]. Other factors associated with PPWR include ethnicity, age, pre-pregnancy BMI, breastfeeding, diet, exercise habit, parity, body image satisfaction, and smoking status [7-9, 14].

Postpartum confinement, commonly known as 'doing the month' or 'sitting the month,' is an East Asian custom that supposedly facilitates recuperation following birth. It is usually practiced for a full month and places several restrictions on the mother's diet and daily routine. Adequate dietary intake is emphasized during this period; however, high-calorie and high-fat foods are usually consumed, which might increase the risk of PPWR. We selected two dishes - sesame oil chicken and braised pork hock, which are often consumed by mothers to facilitate recuperation and improve lactation - as indicators of a fatty diet during this period. Furthermore, in Taiwan, postpartum confinement is traditionally assisted by older family members, but contemporary changes in the social structure have led to the emergence of so-called 'postpartum care centers.' These centers, which are effectively professional service agencies, employ dietitians to design suitable diets for mothers and provide professional attendants to care for newborns while the mothers rest. Because a considerable proportion of Taiwanese women now prefer 'doing the month' in a postpartum care center, we investigated whether variation in postpartum confinement patterns influences PPWR.

Our study examined a cohort of Taiwanese women to investigate their PPWR at 1 and 6 months in addition to the associated factors. The purpose of the study was to identify risk factors and groups at high risk of PPWR in order to develop effective strategies aimed at achieving the optimal PPWR.

\section{Material and Methods}

A cohort study design was adopted to investigate PPWR among women who gave birth at a medical center in northern Taiwan between March 2014 and March 2016. The hospital has approximately 900 beds in its wards, and approximately 3,000 mothers deliver in the hospital annually. Eligible participants were pregnant women aged 18-45 years who gave birth to live infants in the hospital. Women were deemed ineligible if they met any of the following criteria: i) they did not attend prenatal visits at the hospital, ii) the gestational age at delivery was $<35$ weeks, iii) their last prenatal visit was before week 35 , iv) they had a nonsingleton birth, v) they became pregnant again within 6 months following delivery, vi) they had conditions that could affect body weight (e.g., thyroid disease), or vii) they had difficulty reading Chinese. All of the participants signed an informed consent prior to participation, and the study was conducted under the supervision of the Mackay Memorial Hospital Institutional Review Board.

One month following delivery, the participants were required to complete an online questionnaire to obtain the following information: place of residence for the first month, infant feeding methods, frequency of consuming sesame-oil chicken or braised pork hock, weekly exercise time, and body weight (before this pregnancy and 1 month postpartum). Mental health was evaluated using the Edinburgh Postnatal Depression 
Shao et al.: Postpartum Weight Retention Risk Factors in a Taiwanese Cohort Study

Table 1. Baseline participant characteristics and weight retention at follow-up

\begin{tabular}{|c|c|c|c|}
\hline & Mean \pm SD & $\mathrm{N}(\%)$ & Range \\
\hline Maternal age at delivery, years & $33.3 \pm 3.9$ & & $21.0 \sim 45.0$ \\
\hline Gestational age at delivery, weeks & $39.0 \pm 1.1$ & & $35.0 \sim 41.9$ \\
\hline Pre-pregnancy BMI, kg/m² & $21.4 \pm 3.3$ & & $16.5 \sim 40.1$ \\
\hline$<18.5$ & & $66(14.3)$ & \\
\hline $18.5-22.9$ & & $299(64.9)$ & \\
\hline $23-24.9$ & & $46(10.0)$ & \\
\hline$\geq 25$ & & $50(10.9)$ & \\
\hline Gestational weight gain, kg & $12.8 \pm 4.1$ & & $0.2 \sim 35$ \\
\hline Inadequate & & $164(35.6)$ & \\
\hline Adequate & & $200(43.4)$ & \\
\hline Excessive & & $97(21.0)$ & \\
\hline Postpartum weight retention at 1 month, $\mathrm{kg}$ & $4.6 \pm 3.5$ & & $-5 \sim 25$ \\
\hline $\mathrm{Q} 1(<2.0 \mathrm{~kg})$ & & $123(26.7)$ & \\
\hline Q2 (2-4.4 kg) & & $100(21.7)$ & \\
\hline Q3 (4.5-6.9 kg) & & $117(25.4)$ & \\
\hline $\mathrm{Q} 4(\geq 7.0 \mathrm{~kg})$ & & $121(26.3)$ & \\
\hline Postpartum weight retention 6 month, kg & $2.1 \pm 3.3$ & & $-6.5 \sim 15.4$ \\
\hline Q1 $(<0.0 \mathrm{~kg})$ & & $108(23.5)$ & \\
\hline Q2 (0.0-1.9 kg) & & $114(24.7)$ & \\
\hline Q3 (2.0-3.9 kg) & & $115(25.0)$ & \\
\hline $\mathrm{Q} 4(\geq 4.0 \mathrm{~kg})$ & & $124(26.9)$ & \\
\hline \multicolumn{4}{|l|}{ Parity } \\
\hline Primiparous & & $262(56.8)$ & \\
\hline Multiparous & & $199(43.2)$ & \\
\hline GDM, PIH or pre-eclampsia & & $29(6.3)$ & \\
\hline Premature delivery (GA <37) & & $18(3.9)$ & \\
\hline \multicolumn{4}{|l|}{ Mode of delivery } \\
\hline Vaginal & & $371(80.5)$ & \\
\hline C-section & & $90(19.5)$ & \\
\hline 3 rd or 4 th degree perineal lacerations & & $50(10.8)$ & \\
\hline Delivery with wound & & $140(30.4)$ & \\
\hline \multicolumn{4}{|l|}{ Breastfeeding } \\
\hline Exclusive breastfeeding & & $189(41.2)$ & \\
\hline Mixed feeding & & $252(54.9)$ & \\
\hline Exclusive formula-feeding & & $18(3.9)$ & \\
\hline \multicolumn{4}{|l|}{ Place of residence for the 1 st month postpartum } \\
\hline Home & & $222(48.4)$ & \\
\hline Postpartum care center & & $237(51.6)$ & \\
\hline Fatty diet (score $\geq 4)$ & & $54(11.7)$ & \\
\hline Exercise ( $\geq 1 \mathrm{~h} /$ week $)$ & & $85(18.5)$ & \\
\hline EPDS score $(\geq 10)$ & & $143(31.0)$ & \\
\hline EPDS score ( $\geq 13$ ) & & $77(16.7)$ & \\
\hline
\end{tabular}

GDM = Gestational diabetes mellitus; PIH = pregnancy-induced hypertension; GA = gestational age; C-section = cesarean section; Delivery with wound = C-section, 3rd or 4th degree perineal laceration; Mixed feeding = combining of breastfeeding with formula-feeding; EPDS = Edinburgh Postnatal Depression Scale.

Scale (EPDS). Subsequently, their weight was tracked again at 6 months postpartum by email or telephone. Personal information, such as age, parity, measured weight at the last prenatal visit, gestational age at delivery, mode of delivery, degree of perineal laceration after vaginal delivery and gestational complications (such as gestational diabetes mellitus, pregnancy-induced hypertension, and pre-eclampsia), was obtained from medical records.

Pre-pregnancy BMI was classified according to the World Health Organization (WHO) BMI categories for Asians (underweight $<18.5 \mathrm{~kg} / \mathrm{m}^{2}$; overweight $23-25 \mathrm{~kg} / \mathrm{m}^{2}$; or obese $>25 \mathrm{~kg} / \mathrm{m}^{2}$ ). GWG was calculated 
Shao et al.: Postpartum Weight Retention Risk Factors in a Taiwanese Cohort Study

Table 2. Risk factors according to quartiles of 1-month postpartum weight retention

\begin{tabular}{|c|c|c|c|c|c|}
\hline & \multicolumn{4}{|c|}{ Postpartum weight retention at 1 month } & \multirow[t]{2}{*}{ p value } \\
\hline & $\begin{array}{l}\text { Q1 } \\
123\end{array}$ & $\begin{array}{l}\text { Q2 } \\
100\end{array}$ & $\begin{array}{l}\text { Q3 } \\
117\end{array}$ & $\begin{array}{l}\text { Q4 } \\
121\end{array}$ & \\
\hline Maternal age & $33.8 \pm 3.3$ & $33.7 \pm 3.8$ & $32.9 \pm 4.3$ & $32.8 \pm 4.1$ & 0.1165 \\
\hline Gestational age & $38.9 \pm 1.1$ & $39.0 \pm 1.1$ & $38.9 \pm 1.1$ & $39.1 \pm 1.1$ & 0.2383 \\
\hline Prepregnancy BMI & $22.6 \pm 4.2$ & $21.3 \pm 3.3$ & $20.6 \pm 2.6$ & $21.0 \pm 2.6$ & $<0.0001$ \\
\hline$<18.5$ & $13(10.6)$ & $14(14.0)$ & $22(18.6)$ & $17(14.1)$ & 0.0024 \\
\hline $18.5-22.9$ & $67(54.5)$ & $69(69.0)$ & $76(65.0)$ & $87(71.9)$ & \\
\hline $23-24.9$ & $17(13.8)$ & $8(8.0)$ & $11(9.4)$ & $10(8.3)$ & \\
\hline$\geq 25$ & $26(21.1)$ & $9(9.0)$ & $8(6.8)$ & $7(5.8)$ & \\
\hline Gestational weight gain & $9.1 \pm 2.6$ & $11.5 \pm 2.3$ & $13.6 \pm 2.2$ & $16.8 \pm 3.9$ & $<0.0001$ \\
\hline Inadequate & 87 (70.7) & $50(50.0)$ & $21(18.0)$ & $6(5.0)$ & $<0.0001$ \\
\hline Adequate & $30(24.4)$ & $42(42.0)$ & $72(61.5)$ & $56(46.3)$ & \\
\hline Excessive & $6(4.9)$ & $8(8.0)$ & $24(20.5)$ & $59(48.8)$ & \\
\hline Multiparity & $63(51.2)$ & $46(46.0)$ & $47(40.2)$ & $43(35.5)$ & 0.0759 \\
\hline GDM, PIH, or preeclampsia & $9(7.9)$ & $10(10.0)$ & $7(6.0)$ & $3(2.5)$ & 0.1354 \\
\hline Premature delivery & $5(4.1)$ & $3(3.0)$ & $7(6.0)$ & $3(2.5)$ & 0.5264 \\
\hline Delivery with wound & $6(29.3)$ & $24(24.0)$ & $41(35.0)$ & $39(32.2)$ & 0.3345 \\
\hline Exclusive breastfeeding & $55(44.7)$ & $43(43.4)$ & $44(37.6)$ & $47(39.2)$ & 0.6459 \\
\hline Postpartum care center & $69(56.6)$ & $48(48.0)$ & $69(59.5)$ & $51(42.2)$ & 0.0302 \\
\hline Fatty diet & $10(8.1)$ & $14(14.0)$ & $8(6.8)$ & $22(40.7)$ & 0.0221 \\
\hline Exercise & $22(17.9)$ & $17(17.0)$ & $25(21.4)$ & $21(17.4)$ & 0.8195 \\
\hline EPDS score $(\geq 10)$ & $44(35.8)$ & $28(28.0)$ & $30(25.6)$ & 41 (33.9) & 0.2873 \\
\hline
\end{tabular}

GDM = Gestational diabetes mellitus; $\mathrm{PIH}=$ pregnancy-induced hypertension; EPDS = Edinburgh Postnatal Depression Scale.

by subtracting the pre-pregnancy weight from the weight recorded during the last prenatal visit. The GWG estimates were classified according to the 2009 US Institute of Medicine (IOM) guidelines (inadequate, adequate, or excessive). PPWR was calculated by subtracting the pre-pregnancy weight from the postpartum weight; the PPWR at 1 and 6 months was then divided into quartiles. Other classifications assigned to the participants are described as follows: i) Residence in a postpartum care center: those who spent more than 2 weeks in a postpartum care center were assigned to this category. ii) Delivery with wound: women were placed into this category if they had a third- or fourth-degree perineal laceration during vaginal delivery, or if their mode of delivery was cesarean section. iii) Feeding methods: the participants were divided into three groups based on feeding methods: exclusive breastfeeding, exclusive formula feeding, and mixed feeding, which combined breastfeeding and formula feeding. iv) Fatty diet: women were assigned scores according to how frequently they consumed either sesame-oil chicken or braised pork hock in the first month $(0-3$ occasions = 0 points; $4-7$ occasions $=1$ point; $8-15$ occasions $=2$ points; $>15$ occasions $=3$ points). Scores were taken for both foods, and those who received more than 4 points were classified as having a fatty diet. v) Postpartum depression risk: EPDS is a self-report, 10-item questionnaire designed to identify women who have postpartum depression. The total score is 30 , with a score of $10-12$ indicating a moderate risk and $\geq 13$ indicating a high risk.

\section{Statistical Analysis}

SAS version 9.2 (SAS Institute Inc., Cary; NC, USA) was used for all analyses. Data are presented as the mean and standard deviation. Results with $\mathrm{p}<0.05$ were considered statistically significant. Correlations between PPWR and the study variables were examined through a chi-square test and one-way analysis of variance (ANOVA) with Bonfferoni correction. All study variables were included in a multivariate logistic regression analysis comparing the women with higher weight retention (more than the median value) with those with lower weight retention (less than the median value) at 1 and 6 months. 
Table 3. Risk factors according to quartiles of 6-month postpartum weight retention

\begin{tabular}{|c|c|c|c|c|c|}
\hline & \multicolumn{4}{|c|}{ Postpartum weight retention at 6 months } & \multirow[t]{2}{*}{$\mathrm{p}$ value } \\
\hline & $\begin{array}{l}\text { Q1 } \\
108\end{array}$ & $\begin{array}{l}\text { Q2 } \\
114\end{array}$ & $\begin{array}{l}\text { Q3 } \\
115\end{array}$ & $\begin{array}{l}\text { Q4 } \\
124\end{array}$ & \\
\hline Maternal age & $33.6 \pm 3.4$ & $33.6 \pm 4.2$ & $32.7 \pm 3.7$ & $33.3 \pm 4.2$ & 0.2968 \\
\hline Gestational age & $38.9 \pm 1.2$ & $39.0 \pm 1.0$ & $39.0 \pm 1.1$ & $39.1 \pm 1.0$ & 0.6975 \\
\hline Prepregnancy BMI & $22.5 \pm 4.0$ & $21.2 \pm 3.4$ & $20.9 \pm 3.0$ & $21.0 \pm 2.8$ & 0.0009 \\
\hline$<18.5$ & $5(4.6)$ & $23(20.2)$ & $21(18.3)$ & $17(13.7)$ & 0.0032 \\
\hline $18.5-22.9$ & $68(63.0)$ & $66(57.9)$ & $75(65.2)$ & $90(72.6)$ & \\
\hline $23-24.9$ & $16(14.8)$ & $13(11.4)$ & $10(8.7)$ & $7(5.7)$ & \\
\hline$\geq 25$ & $19(17.6)$ & $12(10.5)$ & $9(7.8)$ & $10(8.1)$ & \\
\hline Gestational weight gain & $10.6 \pm 3.1$ & $11.5 \pm 3.6$ & $13.0 \pm 3.1$ & $15.6 \pm 4.3$ & $<0.0001$ \\
\hline Inadequate & $59(54.6)$ & $54(47.4)$ & $35(30.4)$ & $16(12.9)$ & $<0.0001$ \\
\hline Adequate & 37 (34.3) & $46(40.4)$ & $63(54.8)$ & $54(43.6)$ & \\
\hline Excessive & $12(11.1)$ & $14(12.3)$ & $17(14.8)$ & $54(43.6)$ & \\
\hline Multiparity & $58(53.7)$ & $53(46.5)$ & $44(38.3)$ & $44(35.5)$ & 0.0232 \\
\hline GDM, PIH, or preeclampsia & $9(8.3)$ & $6(5.3)$ & $5(5.4)$ & $9(7.3)$ & 0.593 \\
\hline Premature delivery & $6(5.6)$ & $4(3.5)$ & $6(5.2)$ & $2(1.6)$ & 0.3771 \\
\hline Delivery with wound & $34(31.5)$ & $33(29.0)$ & $29(25.2)$ & $44(35.5)$ & 0.3691 \\
\hline Exclusive breastfeeding & $55(50.9)$ & $57(50.0)$ & $42(36.5)$ & $35(28.7)$ & 0.0008 \\
\hline Postpartum care center & $59(55.1)$ & $60(52.6)$ & $66(57.4)$ & $52(42.3)$ & 0.0932 \\
\hline Fatty diet & $9(8.3)$ & $15(13.2)$ & $16(13.9)$ & $14(11.3)$ & 0.576 \\
\hline Exercise & $22(20.4)$ & $25(21.9)$ & $18(15.7)$ & $20(16.1)$ & 0.5269 \\
\hline EPDS score $(\geq 10)$ & $38(35.2)$ & $32(28.1)$ & 36 (31.3) & 37 (29.8) & 0.6998 \\
\hline
\end{tabular}

GDM = Gestational diabetes mellitus; PIH = pregnancy-induced hypertension; EPDS = Edinburgh Postnatal Depression Scale.

\section{Results}

The final cohort comprised 461 women. Table 1 provides an overview of their characteristics. The mean maternal and gestational age at delivery were $33 \pm 3.9$ years and $39 \pm 1.1$ weeks, respectively. Pre-pregnancy BMI was $21.4 \pm 3.3 \mathrm{~kg} / \mathrm{m}^{2}$, with most participants $(64.9 \%)$ having their BMI classified as being within the normal range. Moreover, of all participants, $14.3 \%$ were classified as underweight, $10.0 \%$ as overweight, and $10.9 \%$ as obese. GWG was $12.8 \pm 4.1 \mathrm{~kg}$, with $35.6 \%$ of participants having their GWG classified as inadequate, $43.4 \%$ as adequate, and $21.0 \%$ as excessive according to the 2009 IOM guidelines. The PPWR for the entire cohort was $4.6 \pm 3.5 \mathrm{~kg}$ at 1 month and $2.1 \pm 3.3 \mathrm{~kg}$ at 6 months. The proportion of primiparous mothers $(56.8 \%)$ was marginally higher than that of multiparous mothers, and $19.5 \%$ of deliveries were through cesarean section. The questionnaire survey results revealed that $41.2 \%$ of the participants engaged in exclusive breastfeeding, $51.6 \%$ stayed in a postpartum care center, $11.7 \%$ had a fatty diet, $18.5 \%$ exercised more than $1 \mathrm{~h} /$ week, and $31 \%$ attained an EPDS score of $\geq 10$.

Significant between-groups differences in PPWR were observed at 1 month for GWG, prepregnancy BMI, residence at a postpartum care center, and fatty diet (table 2); and at 6 months for GWG, pre-pregnancy BMI, parity, and exclusive breastfeeding (table 3). Participants with higher GWG tended to have significantly higher PPWR at 1 and 6 months. Specifically, the GWG was $9.1 \pm 2.6 \mathrm{~kg}$ for women with the lowest PPWR (Q1: $<2 \mathrm{~kg}$ ) at 1 month and $16.8 \pm 3.9$ $\mathrm{kg}$ for those with the highest PPWR (Q4: $\geq 7 \mathrm{~kg}$ ). Similarly, the GWG was $10.6 \pm 3.1 \mathrm{~kg}$ for women with the lowest PPWR (Q1: $<0 \mathrm{~kg})$ at 6 months and $15.6 \pm 4.3 \mathrm{~kg}$ for those with the 
Shao et al.: Postpartum Weight Retention Risk Factors in a Taiwanese Cohort Study

Table 4. Logistic regression model for postpartum weight retention

\begin{tabular}{llllll}
\hline & \multicolumn{2}{l}{$\begin{array}{l}\text { Weight retention more than } \\
\text { median at 1 month }\end{array}$} & & \multicolumn{2}{l}{$\begin{array}{l}\text { Weight retention more than } \\
\text { median at 6 months }\end{array}$} \\
\cline { 2 - 3 } \cline { 5 - 6 } & \multicolumn{2}{l}{ crude OR } & adjusted OR & & \multicolumn{2}{l}{$\begin{array}{l}\text { crude OR } \\
\text { crudjusted OR }\end{array}$} \\
\hline Pre-pregnancy BMI & $0.89(0.84-0.95)$ & $0.85(0.77-0.94)$ & & $0.92(0.87-0.98)$ & $0.93(0.87-1.01)$ \\
Gestational weight gain & $1.86(1.66-2.09)$ & $1.92(1.70-2.17)$ & & $1.29(1.21-1.38)$ & $1.30(1.22-1.39)$ \\
Exclusive breastfeeding & $0.79(0.54-1.15)$ & $0.55(0.32-0.94)$ & & $0.48(0.32-0.69)$ & $0.37(0.24-0.58)$ \\
Postpartum care center & $0.92(0.64-1.33)$ & $0.82(0.48-1.40)$ & & $0.84(0.58-1.22)$ & $0.83(0.54-1.28)$ \\
Fatty diet & $1.20(0.68-2.20)$ & $1.23(0.50-3.06)$ & & $1.18(0.67-2.10)$ & $1.17(0.59-2.32)$ \\
Multiparity & $0.81(0.60-1.09)$ & $1.52(0.85-2.71)$ & & $0.75(0.55-1.01)$ & $1.01(0.64-1.61)$ \\
\hline
\end{tabular}

highest PPWR (Q4: $\geq 4 \mathrm{~kg}$ ). Those categorized as obese according to their pre-pregnancy BMI tended to have lower PPWR at both 1 and 6 months. Compared with the primiparous mothers, multiparous mothers tended to have lower PPWR, although the difference became significant only at 6 months. Finally, mothers who engaged in exclusive breastfeeding also tended to have lower PPWR at 6 months.

Multivariate logistic regression analysis was performed to adjust for potential confounders. The variables that remained significantly associated with PPWR were GWG, pre-pregnancy BMI, and exclusive breastfeeding (table 4). Specifically, GWG (adjusted OR: 1.92 (1.70-2.17)), pre-pregnancy BMI (adjusted OR: 0.85 (0.77-0.94)), and exclusive breastfeeding (adjusted OR: $0.55(0.32-0.94))$ were correlated with a 1-month PPWR higher than the median value. Moreover, GWG (adjusted OR: 1.30 (1.22-1.39)) and exclusive breastfeeding (adjusted OR: $0.37(0.24-0.58)$ ) were significantly correlated with a 6-month PPWR higher than the median value.

\section{Discussion}

The present study revealed that women who retained less weight 1 month and 6 months postpartum had gained less weight during pregnancy, had higher BMI before pregnancy, and breastfed exclusively during the first month after delivery.

According to statistics from the Pregnancy Nutrition Surveillance System for the year 2010, excessive GWG is more common among whites (51.9\%) than among Asian/Pacific Islanders (33.5\%) [15]. A retrospective cohort study in Taiwan also reported a low prevalence of excessive GWG (27.3\%) according to IOM guidelines [16]. In our study, the prevalence of excessive GWG was even lower $(21.0 \%)$. Similar to most related studies, our results also show that GWG is a significant predictor of PPWR. A cohort study following up 405 Brazilian women for 9 months postpartum found that those with excessive GWG generally had higher PPWR than those with adequate or inadequate GWG [17]. Overall, the results of the present study accord with those of numerous related studies conducted in Asia, Europe, and America, all of which have indicated that GWG is a major risk factor for PPWR [4-13]. However, the WHO applies different BMI cut-off points to Asians and Caucasians with regard to being overweight (23 and $25 \mathrm{~kg} / \mathrm{m}^{2}$, respectively) or obese (25 and $30 \mathrm{~kg} / \mathrm{m}^{2}$, respectively). Because the IOM guidelines for GWG are based on the WHO standards for Caucasians, it is unclear whether they should also apply to Asians. Therefore, although the present study supports the notion that excessive GWG according to IOM guidelines increases PPWR as well as the association between excessive GWG and gestational complications (which was previ- 
ously recognized by two Asian studies $[16,18])$, further research on the association between Asian GWG and gestational complications, neonatal complications, or neonatal weight is necessary to determine whether IOM guidelines should also apply to Asians.

Our review of the literature revealed contradictory conclusions regarding the association between pre-pregnancy BMI and PPWR $[4,5,7,8,13,17,19,20]$. For example, a study in the US reported that women whose pre-pregnancy BMI indicated that they were overweight or obese were at greater risk of retaining $>9 \mathrm{~kg}$ at 1 year postpartum [5], whereas a study in Sweden suggested that pre-pregnancy BMI is not associated with PPWR [20]. A review article that analyzed the PPWR from 1 month to 15 years noted that, compared with normal-weight women, those who were underweight retained an additional $0.54 \mathrm{~kg}$, whereas those who were overweight and obese had a lower PPWR of -0.81 and $-2.34 \mathrm{~kg}$, respectively [13]. A related study in Taiwan showed that women who are underweight before pregnancy tend to retain more weight after delivery, whereas those who are obese tend to retain less weight after delivery [8]. The findings of the present study suggest that pre-pregnancy BMI correlates negatively with PPWR; in other words, women who are underweight before pregnancy retain more weight after childbirth, which supports the findings of related studies $[7,8,13$, 17]. Similar to having obesity, being underweight has a negative impact on women's health. For underweight women, retaining more weight postpartum may be considered a health benefit because it brings them closer to a normal (i.e., healthy) body weight. Nevertheless, the impact of pre-pregnancy BMI on postpartum weight requires further exploration.

There is a lack of consensus regarding the effects of breastfeeding on PPWR $[5,6,19$, 21-23]. A review published in 2015 indicated that, compared with formula feeding, continual breastfeeding for 3-6 months correlates negatively with PPWR; however, the difference becomes nonsignificant after 6 months [21]. The results of the present study suggest that, compared with exclusive formula feeding or a combination of breast and formula feeding, exclusive breastfeeding for the first month postpartum leads to an earlier return to pre-pregnancy weight at both 1 and 6 months postpartum. Thus, we contend that exclusive breastfeeding has a protective effect on PPWR, which is supported by the findings of several previous studies $[5,6,21,23]$.

The ANOVA results of the present study initially indicated that primiparous women tend to retain more weight than multiparous women at 6 months postpartum; however, this correlation became nonsignificant after adjusting for confounding factors. A review of the literature also indicated that the impact of parity on PPWR remains unclear [6, 24-26]. For example, according to Pidhainy et al. [26], primiparous women tend to exhibit higher GWG and PPWR than multiparous women. They also indicated that primiparous women deliver infants with a lower birthweight than multiparous women for a given weight gain; thus, higher PPWR among primiparous women may be related to characteristics of fat storage. However, clarification of the association between parity and PPWR requires further investigation.

As mentioned previously, we selected two dishes, namely sesame oil chicken and braised pork hock, as indicators of a fatty diet during the postpartum confinement month. Although the ANOVA results indicate that women consuming a fatty diet within 1 month postpartum tend to retain more weight, the regression analysis indicated that this association was nonsignificant, which might be attributable to the lack of representativeness of the selected foods, the influence of other dietary patterns, or potential recall bias in the self-report questionnaire.

An increasing number of women in Taiwan prefer to practice postpartum confinement in care centers. In this study, we investigated whether the site of the postpartum confinement month influences PPWR. Although our multivariable regression results indicate that staying at a postpartum care center is not significantly associated with PPWR, the ANOVA results 
suggest that women who stay at such centers have a lower 1-month PPWR than those who stay at home. This is likely due to the centers providing dietitians who manage the diet of their clients, registered nurses who offer instruction in and assistance with breastfeeding, and facilities for physical exercise, all of which may have contributed to the low PPWR observed in this study. However, because of the lack of related research, the association between residence at a postpartum care center and PPWR requires confirmation by additional studies.

A Brazilian study reported that women who engaged in more physical activity had lower PPWR [6]; this contradicts the findings of Walker et al. [19], who suggested that physical activity is not associated with PPWR. Our study also found no association between exercise and PPWR, which is likely because most of the participants seldom exercising for the first month following delivery. This lack of exercise might be due to the influence of the traditional Asian custom that entails postpartum women avoiding physical activity and resting as much as possible.

The present study had some limitations. First, the participants were all women who gave birth at the same medical center; thus, the cohort might not be nationally representative. Second, pre-pregnancy and postpartum body weight were self-reported and may contain estimation errors; however, a previous study reported that self-reported and measured prepregnancy body weights are strongly correlated [10]. Third, because of the difficulty in accurately measuring GWG (measuring body weight during labor would be infeasible), we calculated GWG by subtracting the pre-pregnancy weight from the weight recorded at the last prenatal visit, as previously described [9]. Finally, because the survey questionnaire was completed only at 1 month postpartum, we could not extend our analysis to 6 months postpartum because of missing information on such factors as infant feeding method, diet, and postnatal depression.

\section{Conclusion}

Excess PPWR is associated with long-term weight gain and is unfavorable to the longterm health of women. Based on the findings of the presentstudy, we recommend the following strategies for limiting PPWR. First, improve patient and staff education regarding the IOM guidelines for GWG and maintain GWG within the adequate range according to patients' BMI. Second, encourage exclusive breastfeeding after childbirth. Finally, health providers should be made aware that women with a relatively low pre-pregnancy BMI are at a high risk of PPWR.

\section{Acknowledgments}

The authors would like to thank all colleagues who contributed to this study. Thanks for helping in data collection, analysis, and commenting on early draft of the work. This work was supported by the Department of Medical Research at MacKay Memorial Hospital/Hospital Research Project, grant no. 103-73.

\section{Disclosure Statement}

The authors declare no conflict of interests. 


\section{References}

1 WHO: Global Health Observatory (GHO) data. Obesity, situation and trends. Geneva, World Health Organization, 2009.

2 Masters RK, Reither EN, Powers DA, Yang YC, Burger AE, Link BG: The impact of obesity on US mortality levels: the importance of age and cohort factors in population estimates. Am J Public Health 2013;103:1895-1901.

3 Atkinson RL, Dietz WH, Foreyt JP, Hill JO, Pi-Sunyer FX, Wing R, Goodwin NJ, Weinsier RL, Hirsch J: Towards prevention of obesity: research directions. Obes Res 1994;2:571-584.

4 Linne Y, Dye L, Barkeling B, Rössner S: Weight development over time in parous women - the SPAWN study - 15 years follow-up. Int J Obes 2003;27:1516-1522.

5 Endres LK, Straub H, Mckinney C, Plunkett B, Minkovitz CS, Schetter CD, Ramey S, Wang C, Hobel C, Raju T, Shalowitz MU: Postpartum weight retention risk factors and relationship to obesity at one year. Obstet Gynecol 2015;125:144-152.

6 Zanotti J, Capp E, Wender MC: Factors associated with postpartum weight retention in a Brazilian cohort. Rev Bras Ginecol Obstet 2015;37:164-171.

7 Cheng HR, Walker LO, Tseng YF, Lin PC: Post-partum weight retention in women in Asia: a systematic review. Obes Rev 2011;12:770-780.

8 Huang TT, Dai FT: Weight retention predictors for Taiwanese women at six-month postpartum. J Nursing Res 2007;15:11-20.

9 Lyu LC, Lo CC, Chen HF, Wang CY, Liu DM: A prospective study of dietary intakes and influential factors from pregnancy to postpartum on maternal weight retention in Taipei, Taiwan. Br J Nutr 2009;102:1828-1837.

10 Kac G, Benicio MH, Velásquez-Meléndez G, Valente JG: Nine months postpartum weight retention predictors for Brazilian women. Public Health Nutr 2004;7:621-628.

11 Mannan M, Doi SA, Mamun AA: Association between weight gain during pregnancy and postpartum weight retention and obesity: a bias-adjusted meta-analysis. Nutr Rev 2013;71:343-352.

12 Huang TT, Wang HS, Dai FT: Effect of pre-pregnancy body size on postpartum weight retention. Midwifery 2010;26:222-231.

13 Rong K, Yu K, Han X, Szeto IM, Qin X, Wang J, Ning Y, Wang P, Ma D: Pre-pregnancy BMI, gestational weight gain and postpartum weight retention: a meta-analysis of observational studies. Public Health Nutr 2015;18: 2172-2182.

14 Melzer K, Schutz Y: Pre-pregnancy and pregnancy predictors of obesity. Int J Obes 2010;34:S44-S52.

15 Centers for Disease Control and Prevention: Pregnancy Nutrition Surveillance, Nation, Summary of Trends in maternal Health indicators by Race/Ethnicity. Table 20D. 2009. https://idph.iowa.gov/Portals/1/Files/ WIC/2010\%20National\%20PNSS\%20tables.pdf (last accessed January 23, 2018).

16 Hung TH, Chen SF, Hsu JJ: Gestational weight gain and risks for adverse perinatal outcomes: a retrospective cohort study based on the 2009 Institute of Medicine guidelines. Taiwan J Obstet Gynecol 2015;54:421-425.

17 Kac G, Benício MH, Velásquez-Meléndez G, Valente JG, Struchiner CJ: Gestational weight gain and prepregnancy weight influence postpartum weight retention in a cohort of Brazilian women. J Nutr 2004;134:661666.

18 Liu Y, Dai W, Dai X, Li Z: Prepregnancy body mass index and gestational weight gain with the outcome of pregnancy: A 13-year study of 292,568 cases in China. Arch Gynecol Obstet 2012;286:905-911.

19 Walker LO, Sterling BS, Kim M, Arheart KL, Timmerman GM: Trajectory of weight changes in the first 6 weeks postpartum. J Obstet Gynecol Neonatal Nurs 2006;35:472-481.

20 Ohlin A, Rössner S: Maternal body weight development after pregnancy. Int J Obes 1990;14:159-173.

21 He X, Zhu M, Hu C, Tao X, Li Y, Wang Q, Liu Y: Breast-feeding and postpartum weight retention: a systematic review and meta-analysis. Public Health Nutr 2015;18:3308-3316.

22 Kramer FM, Stunkard AJ, Marshall KA, McKinney S, Liebschutz J: Breast-feeding reduces maternal lower-body fat. J Am Diet Assoc 1993;93:429-433.

23 Baker JL, Gamborg M, Heitmann BL, Lissner L, Sørensen TI, Rasmussen KM: Breastfeeding reduces postpartum weight retention. Am J Clin Nutr 2008;88:1543-1551.

24 Maddah M, Nikooyeh B: Weight retention from early pregnancy to three years postpartum: a study in Iranian women. Midwifery 2009;25:731-737.

25 Hill B, McPhie S, Skouteris H: The role of parity in gestational weight gain and postpartum weight retention. Women's Health Issues 2016;26:123-129.

26 Lan-Pidhainy X, Nohr EA, Rasmussen KM: Comparison of gestational weight gain-related pregnancy outcomes in American primiparous and multiparous women. Am J Clin Nutr 2013;97:1100-1106. 